\title{
Resource capability modelling for responsive process planning in extended manufacturing facilities
}

\author{
S. M. Ratchev
}

Department of Manufacturing Engineering and Operations Management

University of Nottingham

Nottingham, NG7 2RD, United Kingdom

tel. +44115951 4018, fax. +441159514000

E-Mail: tsvetan.ratchev@nottingham.ac.uk

\begin{abstract}
The paper reports on the development of a decision making methodology for responsive process planning in extended manufacturing facilities using resource capability modelling. The methodology is based on a distributed interactive planning architecture using autonomous decision making agents and data models. The distributed process planning environment and decision making methodology aims to provide the designers and planners with a design support tool for collaborative process plan generation and rapid assessment of the manufacturing feasibility and resource availability at different stages of the product development process.
\end{abstract}

\section{Keywords}

Extended enterprise, process planning, capability modelling 


\section{INTRODUCTION}

The requirements for increased responsiveness to market and the demands for shorter product introduction times and more efficient resource utilisation underline the need for coherent formal approach towards generic resource capability modelling to support the process planning activities. With the advent of "extended" manufacturing facilities capable of reacting to external and internal disturbances by allowing dynamic re-configuration of the manufacturing systems, the process planning activities need to become more responsive and better integrated in the overall decision making process.

Automated process planning strives to bridge the CAD/CAM gap by producing machine-interpretable process plans as inputs to CAM NC systems from the design CAD model of the part (Brooks and Wolf, 1994). Despite the advances in $\mathrm{CAD} / \mathrm{CAM}$ and Concurrent Engineering, however, there is still a limited communication between CAD and CAPP systems (Grabowski and Fu, 1997). The decisions of the designers are not supported by information on machinability which leads to sub-optimum designs. Traditional CAPP systems follow CAD sequentially with the process planning phase starting only after the design phase is completed and the result generated. If some of the design features are not appropriate for machining they can be identified only at process planning stage and the design returned for further changes which costs time and extra resources. A CAD/CAPP interface for complex rotational components is proposed by Jung and Lee (1996) using rotational feature taxonomy and its implementation for data translation between CAD and CAPP using IGES and polygonal decomposition algorithms for feature and geometric precedence recognition.

A collaborative CAD/CAM system which extends a single-location CAD/CAM technology to multi-location application in collaboratively and interactively coediting CAD geometry at a distance using Internet is reported by Kao and Lin (1996). The reported system targets collaborative design, analysis and manufacturing system selection in forming virtual remote machining units.

A multi-agent framework facilitating concurrent product design and planning is proposed by Tan et al. (1996). The framework draws on design-team behaviour in different domains with the goal of providing the infrastructure to enable designers, planners, and managers from different functional areas to collaborate in improving the initial product design. The methodology is based on integrated blackboard architecture with an intelligent agent network using conflict resolution techniques for design refinement and process plan generation. A concurrent engineering framework based on multi-agent planning environment is also reported by Balasubramanian et al. (1996). The approach targets a heterogeneous multi-agent system for concurrent design, manufacturability analysis, process planning, routing 
and scheduling. The above idea is further developed by Gu et al (1997) by integrating design, process planning, and scheduling using a bidding-based approach and STEP based product representation. The proposed methodology assumes an agile manufacturing environment based on intelligent machines capable of making autonomous planning decisions.

A distinctive feature of the process planing in extended manufacturing facilities is that it depends on successful process knowledge management between closely interrelated domains of products, manufacturing processes and geographically distributed manufacturing resources. The specification of resource models to support enterprise engineering has evolved as an active research area during the last decade and a variety of resource models and support architectures have been proposed (Aguiar et al., 1996). A comprehensive review of existing methodologies for process capability representation is also provided by Algeo (1994). Despite the recent developments in the area, there is a need of generic methods to describe the capabilities of manufacturing processes and map the parameters used for their representation to the machines and processing systems participating in the extended manufacturing environment. A vital step in this process is the identification, structuring and representation of process capability knowledge uniquely using a consistent set of generic attributes that can allow several levels of knowledge abstraction and can be used flexibly dependent on the application requirements.

The paper presents a resource capability model and decision making environment developed to support the process planning activities in extended manufacturing facilities. The resource capability model provides unique description of the distribution of processing capabilities and capacities in a manufacturing facility. The model is based upon using multilevel resource models as unique patterns which define the exclusive and shared capability boundaries between different manufacturing resources. The reported research aims to provide the designers with a rapid manufacturing feasibility assessment tool to be used at different design and planning stages in extended manufacturing enterprises.

\section{SYSTEM OVERVIEW}

Process planning in extended manufacturing enterprises is based on selecting production capabilities from different business entities in order to efficiently manufacture a product. It is defined as sequence of steps of transforming component design information into processing requirements and mapping them into manufacturing resources.

In extended manufacturing environment the decision making is based on collaboration between different geographically distributed facilities with a high 
level of autonomy in the extended enterprise organisation. Such autonomy can be effectively described by using a multi-agent representation of the main decision making modules in the process planning system.

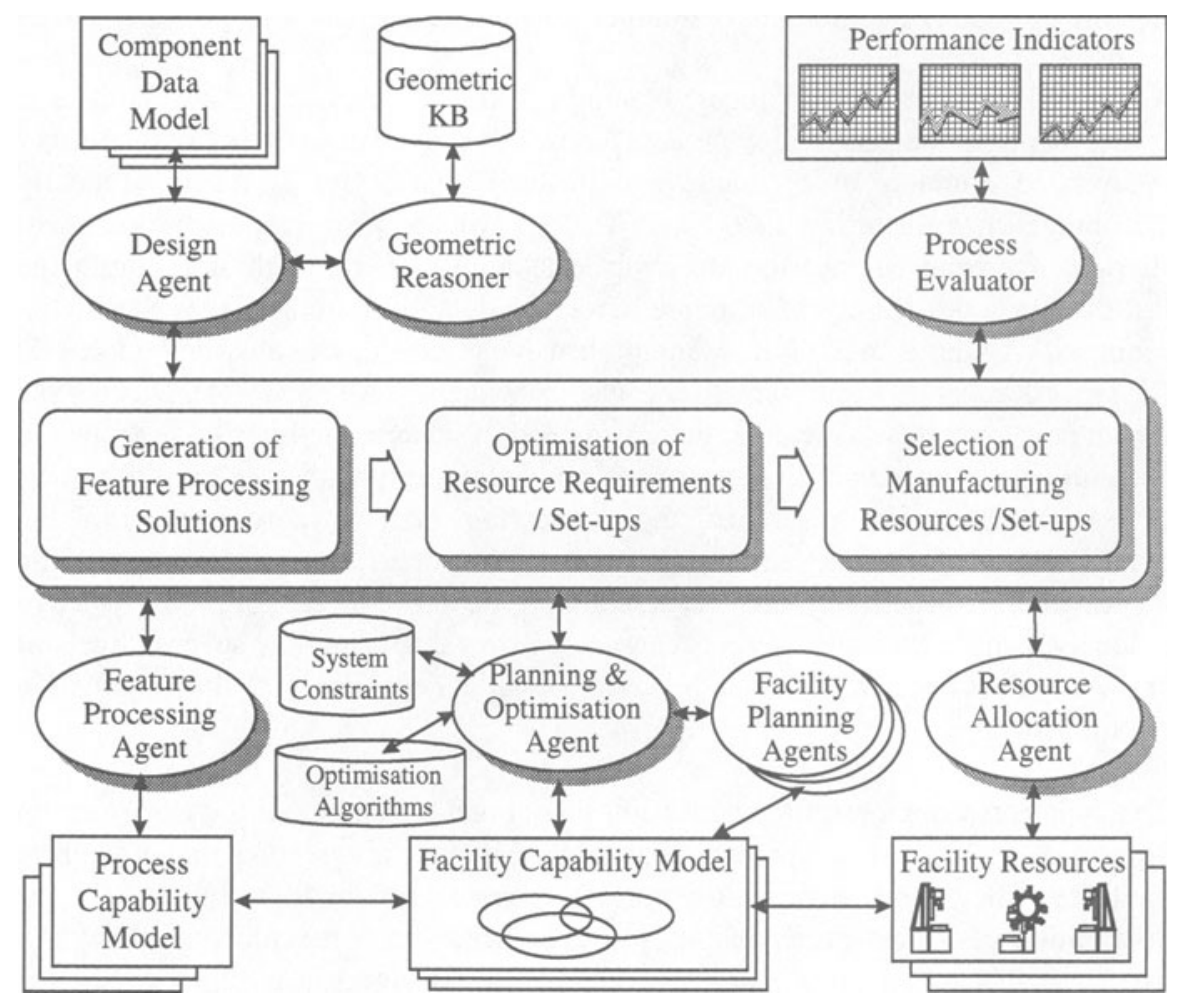

Figure 1 Distributed process planning environment - system overview.

The reported methodology is based on a distributed interactive planning architecture using autonomous decision making agents and a set of object-oriented data models (see Figure 1). The objective is to establish, using a feature based component data model, if the component can be produced using the available for selection system capabilities in the extended manufacturing facility and what capabilities from which business entities would be required to achieve that. The methodology is based on developing a number of autonomous agents (design, geometric reasoning, feature based planning, planning and optimisation, resource selection and process simulation and performance evaluation) for collaborative process planning in extended manufacturing facilities. The system is primarily intended to support a collaborative process plan generation for rapid design evaluation at early product development stages in extended enterprises but can also be used for a variety of other planning tasks. 


\section{PROCESS AND RESOURCE CAPABILITY MODELS}

Manufacturing processes are selected by matching their capabilities to the design requirements. Process capability can be considered at abstract level, independently from any machining facility. It can also be related to specific machine, machine shop, extended manufacturing enterprise. Different levels of process representation are commonly used in different manufacturing tasks, depending on the degree of abstraction required.

\subsection{Process capability model}

The approach adopted in this work is to relate process capability to three fundamental levels. The first is a "form generating schema" to be used for describing process knowledge at a level that is independent of the machine tool and machining facility used for process execution. The second level of abstraction is the "facility" level that is used to describe the distribution of manufacturing capabilities in each facility belonging to the extended enterprise. The third is the "extended enterprise" level which depicts the distribution of manufacturing capabilities between different geographically distributed facilities.

\section{Form generating schemas}

Form generating schema (FGS) is defined as technologically meaningful combination of tool of specific geometry, set of relative motions between a part and the tool, and the typical levels of technological output that can be associated with using that combination of tool and relative motions (see Figure 2). FGS are machine independent, but can be used to provide a generalised description of machine tool capabilities (Gindy and Ratchev, 1997).

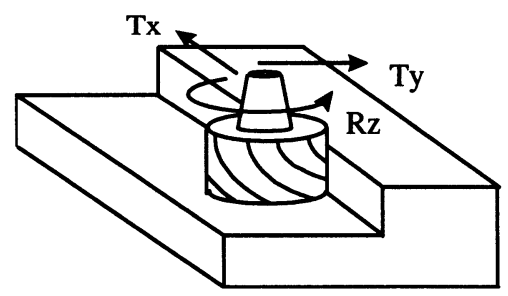

Tool:

Motion Set:

FACE_MILL

Technological Output:

Rz Tx Ty tz

$\{\mathrm{SF}, \mathrm{Tol} ., / /, \ldots\}$

Figure 2 Form generating schema - definition.

\section{Resource Elements (RE)}

Many manufacturing facilities contain identical machines and several machine tools with overlapping capabilities in terms of form generation and technological output. In order to select equipment in process planning, a methodology is needed to compare individual machine capabilities and provide a basis for deciding between resource alternatives before a final machine selection is made. 
The set of machine tools defining a manufacturing facility are described using a set of resource elements (RE) (Ratchev and Gindy, 1997). Each RE represents a collection of form generating schemas which define uniquely the exclusive machine tool capability boundary and the shared boundaries between machine tools (see Figure 3).

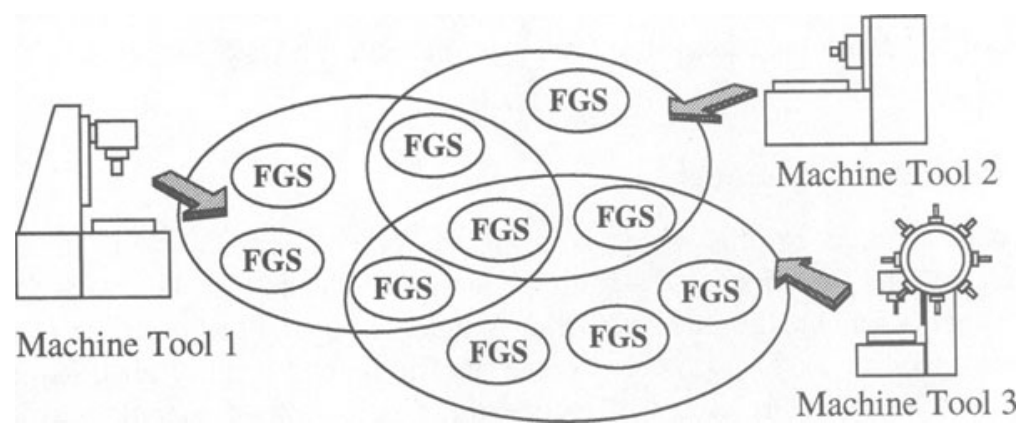

Figure 3 Resource elements - definition.

Resource elements are machining facility specific and capture information relating to the distribution (commonality and uniqueness) of form generating schemas among the machine tools included in the machining facility.

Extended facility representation using technological elements (TE) At extended facility level the processing capability knowledge is described by technological elements (see Figure 4).

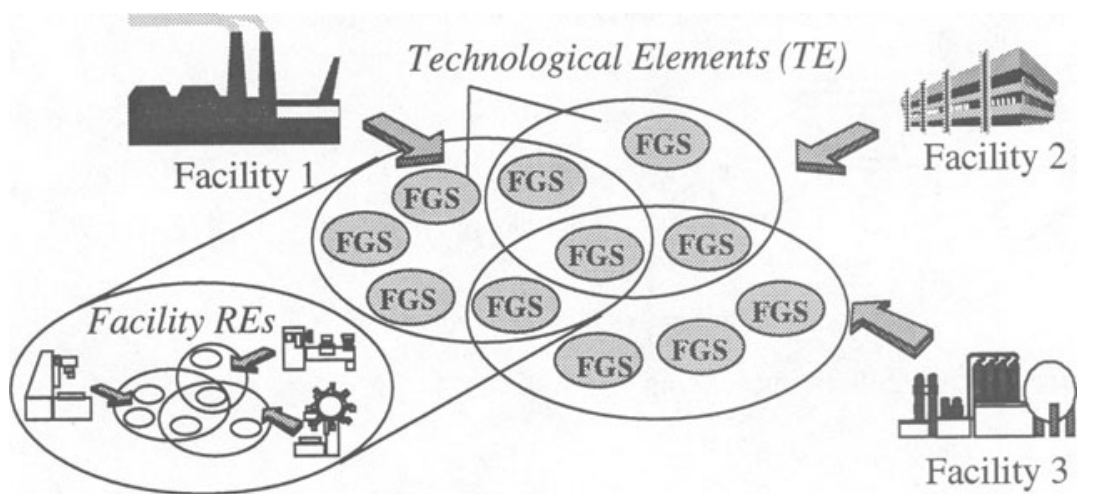

Figure 4 Technological elements - definition.

Technological elements (TE) are collections of form generating schemes which define uniquely the exclusive and shared machining capability boundaries between the machine tools of different entities (Ratchev, 1997). 
Technological elements are extended facility specific and capture information relating to the distribution of FGSs among the machining shops included in the extended enterprise. Although a TE may be attached to several resources, a form generating schema can belong only to one TE. A machining shop is capable of carrying out all the form generating schemas of the TEs associated with it.

\subsection{Extended facility model}

The overall form generating capabilities present in the participating machining facilities are defined as:

$$
F=f_{1}, f_{2}, \ldots, f_{n}
$$

where $f_{k}(k=1,2, . . n)$ are individual form generating schemes, and $\mathrm{F}$ is the full set of FGS available in the extended manufacturing facility M. Each machining facility can be represented by the set of form generating schemes its resources can perform using vector:

$$
M_{k}=m_{1 k}, m_{2 k}, \ldots, m_{n k}, M_{k} \in \mathrm{M}
$$

where

$$
m_{i k}=\left\{\begin{array}{l}
1, F G S f_{i} \text { belongs to machining facility } M_{k}, \\
0, \text { if } F G S f_{i} \text { does not belong to machining facility } M_{k}
\end{array}\right.
$$

The set of TEs describing the overall capability of the extended machining facility is defined by an iterative clustering procedure. Each TE is a group of form generating schemas which appear jointly in each machining facility with the same level of membership:

$$
\text { IF } \forall M_{k} \in \mathrm{M}: m_{p k}=m_{q k}, \text { THEN cluster } f_{p} f_{q} \text { together }
$$

i.e. form generating schemas either appear always together in each machining facility or neither of them is present. The result is a definition of a finite set of TEs describing the distribution of manufacturing resources in the extended machining facility.

A similar procedure is applied to each facility in the extended enterprise to define its constituting resource elements, which depict the unique capability distribution in each individual facility. REs are facility specific and, therefore, will differ between different manufacturing facilities. 


\section{PROCESS PLANNING METHODOLOGY}

An overview of the process planning methodology is provided in Figure 5.

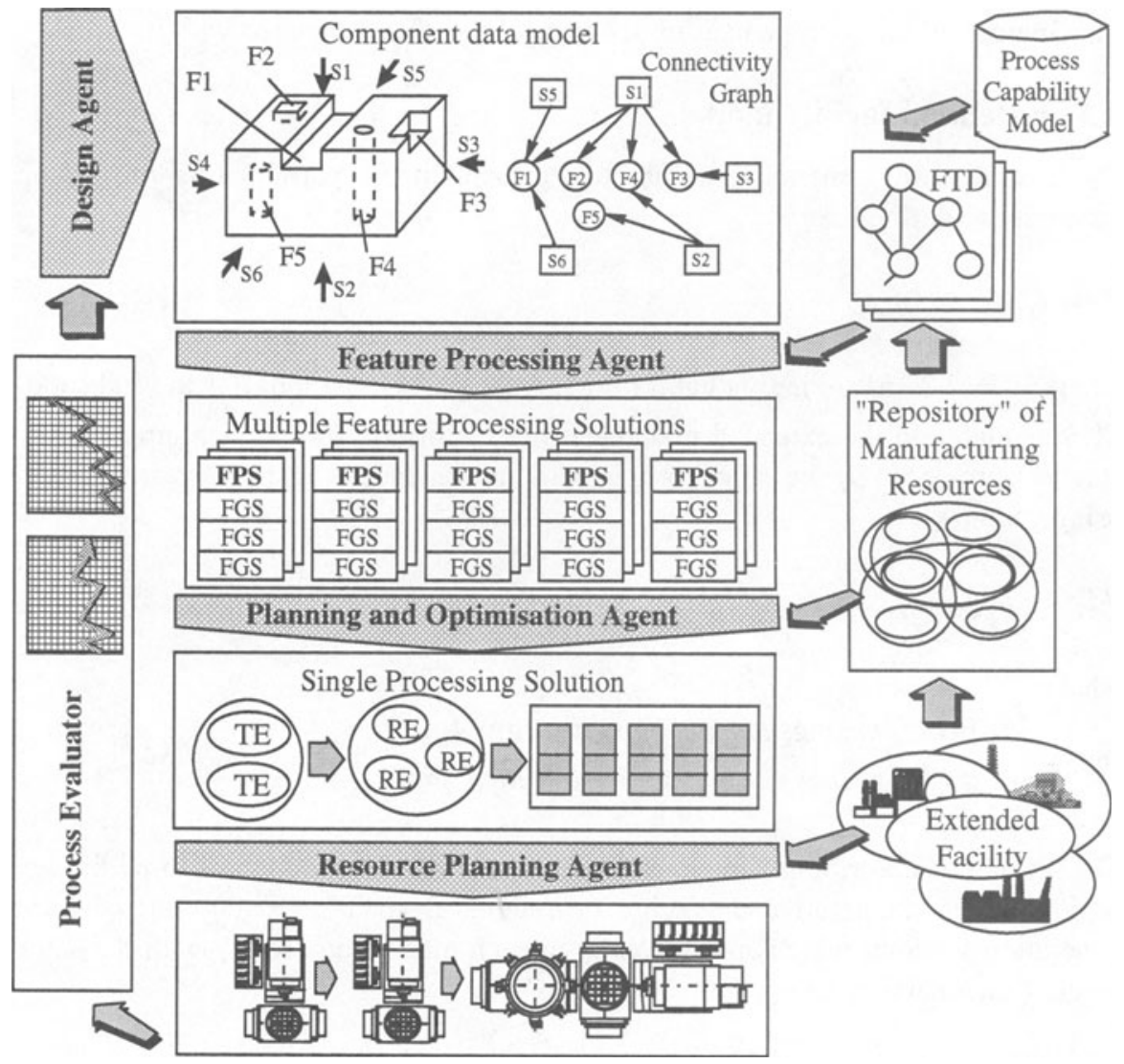

Figure 5 Process planning methodology.

\subsection{Design agent}

The design agent broadcasts the details of the part to the planning and optimisation modules. It provides access to the component data model and handles the communication with the geometric reasoner (refer also to Figure 1). The input to the selection process is provided by component data model based on form features (Gindy and Ratchev, 1991). The relationships between the component features are described by component connectivity graph. Also at component level, the technological relationships (geometrical and positional tolerances) between features are recorded and used in downstream decision making. 


\subsection{Feature processing agent}

Once the types of the features requiring machining and the set of constraints and feature relationships are established, the data model is broadcast to the feature process planner. Using a set of feature transition diagrams processing alternatives are generated and attached to each form feature from each feasible machining approach direction. The process is supported by the process capability model generically describing the available capabilities in the extended facility.

The links between the form generating schemas and the component features are provided through feature transition diagrams (FTD) - collections of form generating schemas capable of producing specific feature geometry with different levels of technological output (Gindy, Ratchev, 1991). Feature processing solutions are generated by finding sets of processes for each feature which can produce the required feature shape and technological output according to the design specifications. Each feature is mapped to a feature transition diagram pointing to a set of company specific process sequences which can be used for producing the feature shape. Feature transition diagrams reflect common extended enterprise specific knowledge on how features are machined.

The process selection is based on finding a set of states in the FTD which match the feature requirements in terms of surface finish and tolerances. All states representing such processes are initialised as final states and the feasible processing sequences are generated as routes connecting the final states to the initial state in the FTD. As a result a set of alternative feature processing solutions (FPS) are attached to each feature from all feasible machining directions.

\subsection{Planing and optimisation agent}

The planning and optimisation agent receives the component data model with the attached feature processing alternatives. As an output of the feature process selection the component processing alternatives are described as sequences of form generating schemas representing the required machining operations in a generalised format (Gindy and Ratchev, 1991). Using the Facility Resource Capability Model each form generating schema is substituted by the corresponding technological element (TE) to which it belongs. The decision making is based on using optimisation agents at two levels - planning and optimisation agent (at extended facility level), and facility planning agents that provide optimisation at participating facilities' level (see Figures 1 and 5).

The optimisation process at extended facility level is designed to decide upon the number and the distribution of TE required for selection of the "best" combination of machining resources in the extended enterprise. The decision making at this level is guided by the need to avoid or, if not possible, to minimise to movement of 
parts between different physical facilities during machining. The algorithm is designed to first establish the exclusive TE required by the part, which can be provided by only one participating facility. The rest of the required resources are outlined by iterative clustering of the shared TEs which are linked to a maximum number of FPSs. Depending on the variety of manufacturing resources available in the extended enterprise and their repetition in different participating facilities several planning alternatives can be generated at this stage.

The planning and optimisation agent communicates with the corresponding facility planning agents for development of the selected planning alternatives at a facility level and establishing the detailed process plan structure.

\section{Facility planning agents}

At a facility level the planning process targets generation of complete component processing solutions within the outlined facility boundaries represented by TEs. The FGS representing the component requirements are transformed into REs which are specific to the facilities represented by the selected set of TEs. A set of system constraints and optimisation criteria are applied to allow selection of the most appropriate processing sequence and set-ups. The selection is based on finding single technological solution per each feature. The process is carried out as iterative removal of the less preferable resource elements (according to a selected system strategy) from the resource element set describing the overall component processing requirements. The removal is conditional upon ensuring that the remaining resource elements are sufficient to fully machine the component.

The result returned from each facility planning agent is a selection of a single processing sequence from single machining direction for each component feature by optimising (usually minimising) the overall number of set ups and required resource elements.

\section{System constraints}

A variety of system constraints are used in selecting the "best" process plan among the process planning alternatives returned by the facility planning agents. The selection constraints are defined with regard to the targeted type of production (e.g. minimum set-ups assumes larger concentration of operations leading to selection of limited number of machines with higher capabilities; maximising the number of set-ups allows selection of wider variety of machines with limited individual capabilities). Component level constraints are also considered so that requirements like "machine in the same set-up" and "machine parallel from the same direction" are taken into account in deciding the set-ups. 


\subsection{Allocation of manufacturing resources}

The final step of the distributed process planning methodology is the selection of the manufacturing (machining) resources to perform the process plan. Again a set of performance criteria are used for optimising the decision making process. Machines are selected using wide range of criteria including: minimum variety of machines; minimum transport movements; maximum utilisation; optimum level of concentration of the operations (e.g. machining centre against dedicated machines), etc.

\subsection{Process plan evaluator}

The process planning methodology is intended as a designers' tool that allows designers to rapidly analyse their designs in terms of manufacturing feasibility and manufacturing system performance. To achieve that the generated process plans need to be evaluated and the results reported back to the design agent. The process plan evaluator is an agent that produces comparative cost estimates by considering the number of set-ups, type of machine tools used, number of transport movements, etc. The evaluation can be further extended to include forecasting of indicators such as: expected lead times, optimum batch sizes, system utilisation, etc.

\section{CONCLUSIONS}

The reported resource capability model provides detailed and accurate description of the distribution of manufacturing capabilities between different facilities participating in an extended manufacturing enterprise and between the resources in each facility. The model facilitates rapid process planning by allowing precise selection of manufacturing resources from different manufacturing facilities. It also supports selection of manufacturing resources at an abstract capability level with the selection of physical resources delayed until later planning stages.

The distributed process planning environment and decision making methodology provide the designers and planners with a design support tool for collaborative process plan generation and rapid assessment of the manufacturing feasibility and resource availability at different stages of the product development process.

\section{REFERENCES}

Aguiar, M.W., Murgatroyd, I.S., Edwards, J.M. (1996) Object-Oriented Resource Models - Their Role in Specifying Components of Integrated Manufacturing Systems, Computer Integrated Manufacturing Systems, 9, 33-48. 
Algeo, M. E. A. (1994) A State of the art survey of methodologies for representing manufacturing process capabilities, NIST Report NISTIR 5391, U.S. Department of Commerce.

Balasubramanian, S., Maturana F. P., Norrie D. H. (1996) Multi-agent planning and coordination for distributed concurrent engineering, International Journal of Cooperative Information Systems, 5, 153-179.

Brooks, S. L., Wolf, M. L. (1994) Overview of Allied Signal's XCUT system, in Advances in Feature Based Manufacturing (ed. J. J. Shah et al.), Elsevier Science B.V., Amsterdam, 399 - 422.

Gindy, N. N. Z., Ratchev, S. M. (1991) Product and machine tools data models for computer aided process planning systems, Computer Applications in Production and Engineering: Integration Aspects (ed. G. Domeingts, J. Browne and $\mathrm{M}$ Tomljanovich ), Elsevier, 527-534.

Gindy, N. N. Z. Ratchev, S. M. (1997) Component grouping for Cellular Decomposition of Manufacturing Facilities using Resource Elements, Integrated Manufacturing Systems, 8, 215 -222.

Grabowski, H., Fu, J. (1997) Integration of CAD/CAPP based on the process planning working space to support design for manufacturing, in Product Modelling for Computer Integrated Design and Manufacture (ed. M. J. Pratt et al.), Chapman \& Hall, London, 418 - 428.

Gu, P., Balasubramanian, S., Norrie D. H. (1997) Bidding-based process planning and scheduling in a multi-agent system, Computers and Industrial Engineering, 32, 1477 - 496.

Jung, M. Y., Lee, K. H. (1996) A CAD/CAPP interface for complex rotational components, International Journal for Production Research, 34, 227-251.

Kao, Y. C., Lin, G. C. (1996), CAD/CAM collaboration and remote machining, Computer Integrated Manufacturing Systems, 9, 149 - 159.

Ratchev, S. M. (1997), Generic Resource Capability Modelling for Formation of Extended Manufacturing Facilities, Proceeding of the 14th International Conference on Production Research, Osaka, Japan, 4-8 August, 18-25.

Tan, G. W., Hayes C. C., Shaw M. (1996) An Intelligent agent framework for concurrent product design and planning, IEEE Transactions on Engineering Management, 43, 297-306.

\section{BIOGRAPHY}

Svetan M Ratchev is a lecturer in the Department of Manufacturing Engineering and Operations Management at the University of Nottingham. He has more than 15 years industrial and research experience in the areas of system design, enterprise capability modelling, and development of integrated collaborative environments for responsive manufacturing. He has published more than 30 research papers and has been involved in a number of national and European research projects. 